\title{
Biotechnology industry responds to gene therapy death
}

The notoriously fickle biotechnology company investment community has hedged its bets on the issue of gene therapy. Following the death last fall of Arizona teenager Jesse Gelsinger and the ensuing federal investigation into this experimental technique, (Nature Med. 6, $6 ; 2000)$, stocks in companies using non-viral vectors, such as San Diegobased Vical, have risen substantially, whereas the share price of firms testing viral vectors has remained steady.

The attractiveness of non-viral vectors is easy to understand in light of Gelsinger's death due to adenovirus toxicity. And the stability of viral vector investments could be based on the notion that industry is confident no further legislation will be imposed on its activities. Or it may be the calm before the storm. Richard Selden, president and CEO of Transkaryotic Therapies, Massachusetts, which conducts ex vivo gene therapy research, speculates, "It is still too early for investors to decide, since the scientific community has yet to come to any solid conclusions about what went wrong."

On the opening day of the Recombinant DNA Advisory Committee's (RAC) meeting in Washington DC to discuss events surrounding Gelsinger's death, the Biotechnology Industry Organization (BIO), which represents
850 companies, academic institutions and state biotechnology centers, issued a statement expressing the industry's belief that gene therapy is already sufficiently strictly regulated and has a good safety record.

In an early attempt to ward off any increased regulatory powers that RAC may be awarded, BIO has "identified several problems with RAC's proposed guidelines" that include an "inappropriate" recommendation for immediate reporting of "serious adverse events unrelated to the treatment;" the creation of "a new format for reporting adverse events that conflicts with current regulations;" and the proposal that reports requested by RAC would contain confidential commercial and patient information.

Instead, BIO proposes that companies voluntarily provide RAC with adverse event reports simultaneously to supplying them to the Food and Drug Administration (FDA). Moreover, it wants RAC and industry to "agree on how the data will be used" in terms of "ensuring patients' privacy rights are protected and companies' trade secrets remain confidential."

However, not all companies are so cautious: Selden believes that "all safety data needs to be made public" because "human health is more important than

confidentiality." He thinks that the chances of a company being harmed by making all safety data available to the public would be so small that it would be worth taking the risk.

Barrie Carter, vice president and director of R\&D at Targeted Genetics (TG), who attended the Washington meeting, says his peers generally agreed that "this was an adenovirus vector event," meaning that those companies involved in gene therapy research using other vectors are in the clear. TG uses the adeno-associated virus (AAV), since unlike adenovirus, AAV is not known to cause disease in humans and does not contain genes that would allow it to induce an immune response. The company issued a press release in November to clarify the distinction between the two vectors, and has trials underway using AAV in cystic fibrosis, from which data should be available later this year.

Valentis' focus is on non-viral gene delivery systems, and although recent events have made Valentis "look closer" at their protocols, they do not foresee the same issues occurring, because their delivery techniques are non-viral.

One company that does use adenovirus vectors is Collateral Therapeutics. Collateral is conducting cardiovascular gene therapy clinical trials using Ad5FGF4-an adenovirus expressing the human growth factor gene FGF4. In fact, there has been one patient death in this trial, but independent evaluation determined that it was unlikely to have been caused by the gene therapy treatment. The FDA has reviewed data from the trial and has not requested or recommended any changes be made to the existing protocol.

\section{Helen Commander, London}

Chronic granulomatous disease ; Cancer; HIV

Genzyme, Cambridge, MA Immune Response, Carlsbad, CA Introgen, Austin, TX

Oxford BioMedica, Oxford, England

Schering Plough, Madison, NJ

Targeted Genetics, Seattle, WA

Transgene, Strasbourg, France

sociated

Haemophilia A; HIV; Cancer

Cardiovascular disease

Cancer; Cystic fibrosis;

Chemoprotection

Cancer; Cystic fibrosis;

Cardiovascular disease

Cystic fibrosis; Gaucher's disease Adenovirus; Retrovirus

Cancer

Cancer

Cancer

Cancer

Cystic fibrosis

Cystic fibrosis; Cancer

Non-viral

Adenovirus

Retrovirus

Adenovirus

Adeno-associated virus

Adenovirus, Adeno-as-

virus

Transkaryotic Therapies, Cambridge, MA Hemophilia A Non-viral

Valentis, Burlingame, CA

Cancer; Cardiovascular disease; Cystic fibrosis

Non-viral

Source: R\&D Insight; ADIS International.
Vical, San Diego, CA

Cancer Non-viral

Cancer Non-viral
Nature Medicine feedback... Questions, comments, complaints? We welcome feedback on the news presented here in the print issue of Nature Medicine and in the "Breaking News" feature on our website (http://medicine.nature.com) Please send your comments on the news (or any other aspect of Nature Medicine) to the Associate Editor, News at k.birmingham@nature.com. 